\title{
THE EFFECTS OF VALPROATE ON HOSTILITY AND IMPULSIVITY AMONG PATIENTS WITH SHIZOPHRENIA
}

\author{
Rancic S. Tosic Golubovic S. Slavkovic V. Sibinovic V. Djordjevic V. Binic I. Antonijevic J. \\ Stojanov J.
}

Objective: It is generally expected that adjunctive valproates can reduce agressive and impulsive behavior. The study compared the specific antiagitation, antihostility and antiimpulsivity effects ot atypical (clozapine, risperidone) or typical (haloperidol) antipsychotic therapy with valproate adjuntive treatment to mentioned antipsychotics among schizophrenic, schizophreniform or schizoaffective pateinets experiencing an acute psychotic episode.

Methods: Improvement in agitation on BPRS, agitation, impulsivity and hostility score of PANSS were the principal outcome measures and covariates included positive symptoms of schizophrenia. Treatment efficacy and side effects were observed by using CGI. Evaluation process was preformed at the admission, after first, second third and fourth week of treatment.
Results: Adjunctive treatment with valproate was associate with statistical significant lower scores on the BPRS agitation score and PANSS impulsivity and hostility items compared with antipsychotic monotherapy, even after the first week of treatment. The effect on agitation, hostility and impulsivity was statistically independent of antipsychotic effect on other PANSS items reflecting positive symptoms of schizophrenia.

Conclusions: Reduction of the agitation, impulsive and hostile behavior occured faster and was superior during valproate adjunctive therapy. This reduction was independent of the effect on other positive psychotic symptoms.
Key word: schizophrenia, mood stabilizers, valproate, carbamazepine, aggitation, hostility.
Reference:

1. Afaq I, Riaz J, Sedky K, et al. Divalproex as a calmative andjunct for aggressive schizophrenic patients. Journal of the Kentacky Medical Association 2002;100(1): 17-22.

2. Simhandl Ch, Mesyaros K. The use of carbamazepine in the treatment of schizophrenic and schizoaffective psychosis: A revieww. J Psychiatr Neurosci 1992;17:1-14. 